\title{
Working memory deficits in multiple sclerosis: a controlled study with auditory P600 correlates
}

\author{
C Sfagos, C C Papageorgiou, K K Kosma, E Kodopadelis, N K Uzunoglu, \\ D Vassilopoulos, A D Rabavilas
}

J Neurol Neurosurg Psychiatry 2003;74:1231-1235

See end of article for authors' affiliations

Correspondence to

Dr C C Papageorgiou,

Department of Psychiatry,

Eginition Hospital,

University of Athens,

74 Vas Sophias Avenue,

Athens, Greece;

panchris@otenet.gr

Received

3 December 2002

Accepted in final revised

form 26 March 2003

\begin{abstract}
Background: Recently, the P600 component of event related potentials, a waveform that is conceived to be generated and/or modulated by basal ganglia and cingulate area has been considered an index of the completion of any synchronised operation after target detection, having much in common with working memory operation. Moreover, dysfunction of these brain structures as well as working memory deficits have been implicated in the pathophysiology of multiple sclerosis. The aim of this study was to investigate the patterns of P600 elicited during a working memory test in multiple sclerosis patients compared with healthy controls.

Methods: Twenty two definite, chronic progressive multiple sclerosis patients, with recent exacerbation of their illness, and 20 normal subjects matched for age, sex, and educational level, were studied with a computerised version of the digit span test of Wechsler batteries. Auditory P600 were measured during the anticipatory period of this test.

Results: The patient group, as compared with healthy controls, showed significantly reduced latencies of P600 at left frontal areas and reduced P600 amplitudes at left temporoparietal region. Moreover, memory performance of patients was significantly more impaired when compared with healthy controls.

Conclusions: These findings may indicate that multiple sclerosis is associated with abnormal features of the completion of synchronised operation after target detection, as they are reflected by P600 amplitudes and latencies. Dysfunction of this mechanism may contribute to the identification of basic cognitive processes that could account for the cognitive deficits in multiple sclerosis.
\end{abstract}

C ognitive impairment is a common clinical feature of multiple sclerosis (MS), occurring in up to $65 \%$ of patients with this disorder. ${ }^{12}$ Repeatedly, it has been suggested that the cognitive impairment in MS patients is strongly associated with limitations in work and social activities. $^{3}$

Recent neuropsychological studies provided evidence indicating that working memory (WM) deficits may be involved in MS. ${ }^{45}$ However, brain mechanisms underlying these deficits continue to be a subject of ongoing investigation, as their patterning and specificity still remain unclear. This is particularly evident in psychophysiological research. The existing evidence indicates that the multifocal demyelination of MS leads to a disruption of the multiple interconnected brain areas, which form the substrate of the working memory. ${ }^{5-7}$

Event related potentials (ERPs) provide a valuable means for studying brain-behaviour relations. ${ }^{8}$

Pelosi et al, recording both auditory and visual ERPs during the memorising as well as the recognition and matching of digits of a short-term memory paradigm, found that early MS patients as compared with healthy controls displayed a delay of the N270/N290 waves and a decreased amplitude of the major positivities (P400/P560). ${ }^{7}$ Although these abnormalities have been referred to with regard to both modalities, abnormal changes were more noticeable in the auditory modality. The delay of the negative waves has been interpreted as "an index of slowed processing between stimulus presentation and motor response selection", while the reduction of the amplitudes as "a manifestation of either the allocation of attentional resources to cope with the increasing task demands or a desynchronization of decision making and/or response selection and execution". It should be noted that in this study only three leads were used, namely Fz, Cz, and Pz.
The P600 component of ERPs or late positive potentials (elicited between 500 and $800 \mathrm{~ms}$ or later after warning stimuli) is accepted as reflecting the completion of any synchronised operation immediately after target detection. In other words, it signals "the second pass parsing processes" of information processing. Specifically, its amplitude is considered as an index of the cost of reprocessing, while its latency as a function of onset and duration of parsing processes. ${ }^{9}{ }^{10}$ Furthermore, it has been suggested that the P600 component may be related to WM. ${ }^{1}$

Contemporary neuropsychological views define working memory as the capacity to keep information on line as necessary for an ongoing task. ${ }^{12}{ }^{13}$ According to this prospect WM comprises those functional components of cognition that allow humans to comprehend and mentally represent their immediate environment, to retain information about their past experience, to support the acquisition of new knowledge, to solve a problem, as well as to formulate, relate, and act on current goals. In other words WM is not for "memorising" in itself. It is rather in the service of complex cognitive activities, such as reasoning, problem solving, and decision making. ${ }^{14}$

Concerning the anatomical locus of neural activity underlying the generation of P600, intracranial recordings suggest that P600 is associated with activity in wide ranged brain structures including frontal, temporal, and superior parietal regions, which are believed to contribute significantly to some aspect of information processing during recognition memory. ${ }^{16}$ Additionally, the results of more recent research indicate that the P600 waveform is generated and/or

Abbreviations: EDSS, Expanded Disability Status Scale; ERP, event related potentials; MS, multiple sclerosis; WM, working memory 
Table 1 Sample characteristics: demographic variables (age, education, duration of illness), clinical characteristics from the EDSS, as well as memory performance (mean (SD) and statistical significance) *

\begin{tabular}{|c|c|c|c|c|c|}
\hline & \multicolumn{2}{|c|}{$\operatorname{MSP}(n=22)$} & \multicolumn{2}{|c|}{ Controls $(n=2)$} & \multirow[b]{2}{*}{$\mathrm{p} t$ test } \\
\hline & Mean & (SD) & Mean & (SD) & \\
\hline Age (y) & 40.77 & 9.38 & 38.7 & 7.1 & NS \\
\hline Education (y) & 13.36 & 2.53 & 12.85 & 2.15 & \\
\hline Duration of illness (y) & 9.31 & 9.62 & & & \\
\hline Memory performance & 51.31 & 21.30 & 67.4 & 6.77 & 0.002 \\
\hline Duration of illness & 9.31 & 9.62 & & & \\
\hline Pyramidal function & 2.77 & 1.44 & & & \\
\hline Cerebellar function & 2 & 1.77 & & & \\
\hline Brain stem function & 0.95 & 1.29 & & & \\
\hline Mental function & 0.54 & 0.73 & & & \\
\hline Sensory function & 1.54 & 1.56 & & & \\
\hline Urinary bladder function & 0.36 & 0.65 & & & \\
\hline Visual-optic function & 0.40 & 1.22 & & & \\
\hline Total score & 4.29 & 1.92 & & & \\
\hline
\end{tabular}

modulated by both cingulate gyrus ${ }^{11}$ and basal ganglia. ${ }^{10}$ Furthermore, current knowledge based on neuroimaging studies suggests a key role of cingulate area $^{17}$ as well as basal ganglia $^{18}$ in the pathophysiology of MS.

The foregoing points motivated us to examine aspects of "second pass parsing" processes of information processing as are reflected by $\mathrm{P} 600$ elicited during a WM test in MS patients compared with those of healthy controls.

\section{METHODS}

\section{Participants}

Twenty two definite, chronic progressive MS patients (8 men, and 14 women), with recent exacerbation of their illness participated in the study. They were hospitalised in the neurology clinic of the Athens University Medical School or had been previously hospitalised and were being followed up. Patients were selected according to the Poser $e^{2} \mathrm{al}^{19}$ criteria for definite or probable MS, and had no history of alcohol/drug misuse, head injury, or stroke. Fourteen of the patients had a relapsing remitting form and the rest had a progressive form of MS. All patients were assessed with the Kurtzke Expanded Disability Status Scale ${ }^{20}$ (EDSS see table 1).

Twenty controls were recruited from the hospital staff and local volunteer groups. They were free of psychiatric and physical illness. All participants were right handed as assessed by the Edinburgh Inventory, ${ }^{21}$ and had no history of any neurological or hearing problems. Written informed consent was obtained from both patients and control subjects.

\section{Stimuli and procedure}

Patients and controls were evaluated by a computerised version of the digit span Wechsler test. ${ }^{22}{ }^{23}$ Although the digit span of the Wechsler test is considered a test of short-term memory, more recent reports emphasise its relevance to $W_{M} .^{24}{ }^{25}$ The subjects sat in an anatomical chair placed inside an electromagnetically shielded room. An outline of the procedure is provided in figure 1 . A single sound of either high $(3000 \mathrm{~Hz})$ or low frequency $(500 \mathrm{~Hz})$ was presented to the subjects, who were asked to memorise the numbers that followed. The warning stimulus lasted $100 \mathrm{~ms}$. A one second interval followed and then the numbers to be memorised were presented. At the end of the number sequence presentation, the signal tone was repeated and subjects were asked to recall the administered numbers as quickly as possible. The numbers were recalled by the subject in the same (low frequency tone) or in the opposite order (high frequency tone) than that presented to the participant.

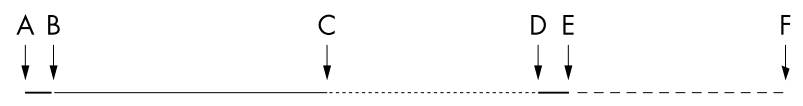

\begin{tabular}{|l|l|}
\hline Time period & 1.1.1.1 Action \\
\hline$A B(100 \mathrm{~ms})$ & $\begin{array}{l}\text { Administration of warning stimulus (500 or } \\
3000 \mathrm{~Hz}, 65 \mathrm{~dB}) .\end{array}$ \\
\hline $\mathrm{AC}(1100 \mathrm{msec})$ & Recording of ERPs. \\
\hline $\mathrm{CD}$ (varies) & $\begin{array}{l}\text { Computerised administration of the set of } \\
\text { numbers of the Wechsler Direct Auditory } \\
\text { Memory Test. The duration of this period varies } \\
\text { depending upon the numbers of digits to be } \\
\text { recalled in each trial (from two to nine digits } \\
\text { across trials). However, between administered } \\
\text { digits, the time interval is one second. }\end{array}$ \\
\hline DE (100 ms) & Repetition of the warning stimulus \\
\hline $\begin{array}{l}\text { EF (varies } \\
\text { between } 15 \\
\text { and } 30 \mathrm{~s})\end{array}$ & $\begin{array}{l}\text { Recording of the memory recall performance } \\
\text { according to the accuracy of responses }\end{array}$ \\
\hline
\end{tabular}

Figure 1 Outline of the experimental procedure.

Before any recording of ERPs, a pre-process was performed so that the two sounds were differentiated by the subjects. According to this process, various trials have taken place until each subject understood both the different tonalities and the requirements of the test, concerning the storage and retrieval of presented numbers. After the completion of the above mentioned process, a rest period of five minutes followed, before the recording of the ERPs.

ERPs were recorded during the $1100 \mathrm{~ms}$ interval between the warning stimulus and the first administered number. The electrophysiological signals were recorded through $\mathrm{Ag} / \mathrm{AgCl}$ electrodes. Electrode resistance was kept constantly below 5 kOhm. EEG activity was recorded from 15 scalp electrodes based on the International 10-20 system of electroencephalography, ${ }^{26}$ referred to both earlobes. An electrode placed on the subject's forehead served as ground. The bandwidth of the amplifiers was set at $0.05 \mathrm{~Hz}$ to $35 \mathrm{~Hz}$. During the administration of stimuli, the subjects had their eyes closed to minimise eye movements and blinks. Eye movements were recorded through electro-oculogram (EOG) and recordings with EOG higher than $75 \mathrm{~V}$ were rejected.

Warning stimuli, as well as learning material-that is, the numbers to recall-were presented binaurally via earphones at an intensity of $65 \mathrm{~dB}$ sound pressure level. The evoked biopotential signal was submitted to an analogue to digital conversion, at a sampling rate of $500 \mathrm{~Hz}$, and was averaged by a computerised system. Each recording session consisted of 26 repetitions of the trial. Eye movements corresponding to EOG higher than $75 \mu \mathrm{V}$, resulting in rejection of the recording, ranged from one to two per investigation. Thus the minimum number of artefact free trials that were averaged to produce an ERP, was 24.

As the warning stimuli were of two different frequencies, one high and one low, it was not clear whether they could generate the same P600, although the P600 component is included in the array of late endogenous ERPs' components, which normally are not modality specific. ${ }^{8}$ To ensure that there were no differences in the P600 waveforms caused by frequency modalities, we conducted $t$ test series comparisons between the P600 waveforms (amplitudes and latencies) evoked by the two frequency modalities ( 13 high and 13 low frequencies), in all subjects. No differences were found in the P600 waveforms, by frequency, in each subject. 

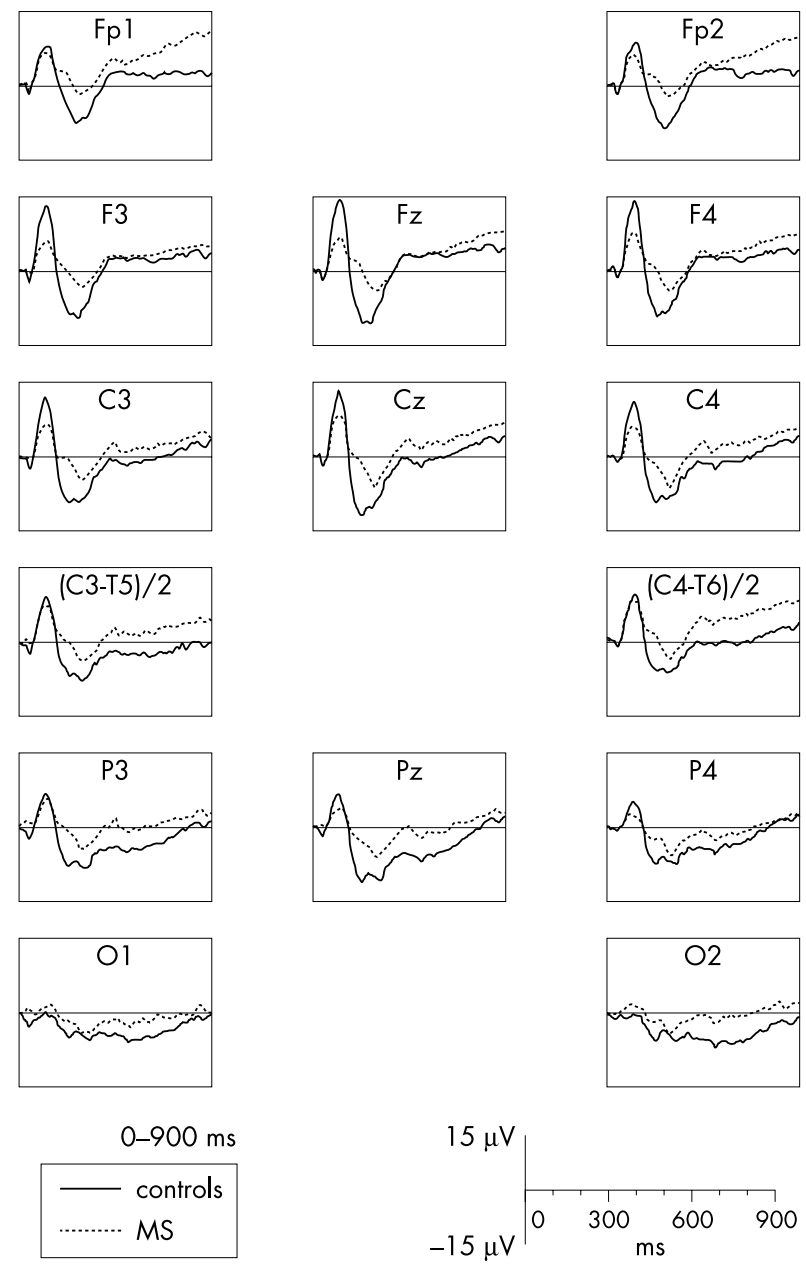

Figure 2 Waveforms' graphical display.

Consequently the pooled P600 waveforms for each lead (fig 2 ) were used in the analysis. The following parameters were calculated:

(A) ERPs were recorded for each subject at leads Fpl, Fp2, F3, F4, C3, C4, C3-T5/2, C4-T6/2, P3, P4, O1, O2, Pz, Cz, and Fz for each of the 26 test repetition. In this context, it should be noted that the positions C3-T5/2 and C4-T6/2 are used as electrode leads, because these positions correspond to brain areas serving verbal memory and language. ${ }^{27}$ Recordings with acceptable EOG level were averaged, for each lead, by a computerised system. An algorithm was used, which identified the P600 as the most positive peak, in each averaged lead curve, between 500 and $800 \mathrm{~ms}$, after the warning stimulus. Peak amplitudes were measured relative to the mean amplitude of the $100 \mathrm{~ms}$ prestimulus baseline period and latency measurements were computed relative to stimulus onset.

(B) The behavioural performance refers to the number of recalled digits. It should be noted that the total digits presented were 149 .

\section{Statistical analysis}

The statistical analysis used for each group of variables (amplitudes and latencies) was exclusively dictated by the normality of their distributions. Thus the KolmogorovSmirnov goodness of fit test shows that both the amplitudes and the latencies are consistent with a normal distribution, as well as a multivariate normal distribution, while the equality of the covariance matrices was ascertained with Box's M test. This permitted the use of multivariate tests for the examination of the overall group differences, while group differences
Table 2 Mean (SD) latencies $(\mu \mathrm{V})$ of the P600 waveform for the two groups, at each lead

\begin{tabular}{|c|c|c|c|c|c|c|}
\hline & \multicolumn{2}{|c|}{$M S P(n=22)$} & \multicolumn{4}{|c|}{ Controls $(n=20)$} \\
\hline & Mean & (SD) & Mean & (SD) & F 1,40 & $\eta 2$ \\
\hline Fpl & 557.1 & 58.9 & 675 & 91.9 & 24.91 ** & 0.384 \\
\hline F3 & 576 & 59.7 & 664.9 & 101.5 & $12.23^{* *}$ & 0.234 \\
\hline C3-T5/2 & 599.0 & 70.7 & 628.1 & 86.0 & 1,43 & 0.035 \\
\hline $\mathrm{C} 3$ & 579.2 & 62.9 & 621.6 & 89.3 & 3.20 & 0.074 \\
\hline Fp2 & 586.3 & 69.2 & 663.1 & 84.5 & $10.44^{* *}$ & 0.207 \\
\hline $\mathrm{F} 4$ & 597.7 & 78.2 & 643.5 & 84.5 & 3.32 & 0.077 \\
\hline C4-T6/2 & 610.3 & 87.3 & 596.9 & 89.4 & 0.24 & 0.006 \\
\hline $\mathrm{C} 4$ & 605.6 & 83.7 & 582 & 87.7 & 0.80 & 0.020 \\
\hline O1 & 585.1 & 89.7 & 569.3 & 52.9 & 0.48 & 0.012 \\
\hline $\mathrm{O} 2$ & 589.1 & 100.1 & 583.1 & 70.3 & 0.05 & 0.001 \\
\hline P4 & 583.2 & 86.7 & 568 & 63.9 & 0.41 & 0.010 \\
\hline P3 & 621.8 & 110.4 & 587.1 & 75.3 & 1.39 & 0.034 \\
\hline $\mathrm{Pz}$ & 584.3 & 75.0 & 587.8 & 82.6 & 0.02 & 0.000 \\
\hline $\mathrm{Cz}$ & 599.3 & 84.6 & 604.5 & 92.1 & 0.04 & 0.001 \\
\hline $\mathrm{Fz}$ & 584.0 & 64.6 & 635.3 & 104.4 & 3.72 & 0.085 \\
\hline
\end{tabular}

Two asterisks next to the $F$ value denote statistically significant differences between the two groups at the $1 \%$ level.

for each lead individually was ascertained by the corresponding univariate statistics as well as by stepdown procedures. At each step the most important leads played the part of covariates. In the case of latencies stepwise discriminant analysis was also applied to elucidate the discrimination power of the specific group of variables-that is, whether they would be able to predict group membership.

One way analysis of variance was used to assess group differences in memory performance measures. Results were considered significant at the 0.05 level. Finally, linear regression models were applied for the elucidation of the effect of clinical characteristics and the memory performance on the P600 waveforms differences.

\section{RESULTS}

\section{Comparison of $\mathrm{P} 600$ in MS patients and controls}

Table 2 shows the mean latencies and standard deviations in milliseconds of the P600 waveform for the two groups at each lead. Both Pillai's and Hotelling's trace manifested statistically significant differences between the overall means of the compared groups $(p=0.021)$, while the value of Wilk's $\eta(0.412)$ shows that $58.8 \%$ of the overall variability can be attributed to group differences. Univariate $F$ tests showed that between group variability significantly exceeded the within group variability $(p<0.01)$ at leads Fp1, F3, and Fp2. Estimates of effect size, especially for Fpl $\left(\eta^{2}=0.384\right)$ are quite high. Stepdown procedures reduce the number of leads where significant differences between the groups are observed to leads Fpl and F3. Likewise stepwise discriminant analysis showed the two above mentioned leads were able to correctly classify $85.7 \%$ of the original grouped cases ( 16 of 20 normal subjects and 20 of 22 of the MS patients).

Table 3 shows the mean amplitudes and standard deviations in microvolts of the P600 waveform for the compared groups at each lead. It is interesting to note that neither Pillai's nor Hotelling's trace (0.472 and 0.893 respectively) manifested statistically significant differences between the overall means of the compared groups, while the value of Wilk's $\eta(0.528)$ shows that only $47.2 \%$ of the overall variability can be attributed to group differences. Nevertheless, univariate $F$ tests showed that there are significant group differences at the 0.05 level at leads C3-T5/2, C4-F6/2, C4, O1,O2, P3, Pz, and Cz. However, $\eta^{2}$ values show only medium effect size, while stepdown procedures reduce the significance to only one lead, namely C3-T5/2. 
Table 3 Mean (SD) amplitudes (ms) of the P600 waveforms for the two groups at each lead

\begin{tabular}{|c|c|c|c|c|c|c|}
\hline & \multicolumn{2}{|c|}{$M S P(n=22)$} & \multicolumn{4}{|c|}{ Controls $(n=20)$} \\
\hline & Mean & (SD) & Mean & (SD) & $F 1,40$ & $\eta 2$ \\
\hline $\mathrm{Fpl}$ & -2.45 & 7.8 & 1.45 & 6.0 & 3.213 & 0.074 \\
\hline F3 & -0.34 & 5.6 & 1.30 & 5.1 & 0.972 & 0.024 \\
\hline C3-T5/2 & 1.33 & 4.2 & 5.76 & 4.9 & 9.740 ** & 0.196 \\
\hline C3 & 1.85 & 3.4 & 4.24 & 4.9 & 3.312 & 0.076 \\
\hline Fp2 & -1.81 & 6.8 & 1.13 & 6.1 & 2.158 & 0.051 \\
\hline $\mathrm{F} 4$ & -0.25 & 5.6 & 1.01 & 4.6 & 0.625 & 0.015 \\
\hline $\mathrm{C} 4-\mathrm{T} 6 / 2$ & -1.00 & 6.6 & 3.51 & 5.0 & $6.054^{*}$ & 0.131 \\
\hline $\mathrm{C} 4$ & 1.17 & 4.4 & 4.51 & 4.7 & 5.498 * & 0.121 \\
\hline 01 & 5.32 & 4.3 & 8.59 & 5.0 & $5.047^{*}$ & 0.112 \\
\hline $\mathrm{O} 2$ & 4.80 & 5.1 & 9.93 & 5.8 & $9.112 * *$ & 0.186 \\
\hline P4 & 4.78 & 4.5 & 7.63 & 5.2 & 3.584 & 0.082 \\
\hline P3 & 3.78 & 3.7 & 7.53 & 4.4 & 8.783 * * & 0.180 \\
\hline $\mathrm{Pz}$ & 4.09 & 5.1 & 8.919 & 5.7 & $8.275^{\text {** }}$ & 0.171 \\
\hline $\mathrm{Cz}$ & 0.63 & 4.8 & 4.03 & 4.2 & $5.644^{*}$ & 0.124 \\
\hline $\mathrm{Fz}$ & -0.77 & 6.6 & 0.48 & 4.5 & 0.498 & 0.012 \\
\hline
\end{tabular}

An asterisk next to the $F$ value denotes statistically significant differences between the two groups at the $5 \%$ level, two asterisks, at the $1 \%$ level.

\section{Behavioural data}

The analysis revealed that the patient group had significantly attenuated memory performance as compared with healthy controls $\left(F_{1,40}=10.41, \mathrm{p}<0.01\right)$.

\section{Correlation of P600 with the clinical characteristics and memory performance}

Stepwise regression analyses, in which the clinical characteristics and the memory performance were set as the independent variables and observed differences regarding the P600 waveforms were the dependent variable, showed: firstly, that duration of illness was positively associated with P600 latencies at F3 site $\left(\beta=0.539, t_{20}=2.860, p=0.010\right)$. Secondly, cerebellar clinical dimension was negatively related to P600 latencies at Fpl site $\left(\beta=-0.504, t_{20}=-2.609, p=0.017\right)$.

\section{DISCUSSION}

The aim of this study was to investigate aspects of information processing in MS patients as reflected by P600 waveform when compared with those of healthy controls. The results showed that the patient group demonstrated significantly shortened latencies of $\mathrm{P} 600$ waveform at Fpl and F3 leads, as well as reduced amplitudes at C3-T5/2 lead.

The importance of the observed differences concerning the P600latencies maybebetter explored ifweconsiderboth psychophysiological and neurobiological views regarding this ERP component, which was mentioned earlier.

Thus, the patterns of the results obtained in this study suggest that patients with MS demonstrated impairments in the stage of information processing that "assigns a specific response to a specific stimulus" 28 as they are reflected by the P600 latencies elicited during a WM test.

It is noteworthy that the obtained differences with regard to the P600 latencies at left prefrontal region were associated negatively with the degree of the cerebellar impairment.

This finding suggests that the cerebellum influences second pass parsing defects in MSP. This suggestion is compatible with convergent results of very recent studies indicating the involvement of both brain structures in WM and time keeping operations. ${ }^{29-31}$

In addition, this study yielded a positive association between the duration of illness and the latency of P600 recorded at left dorsal frontal region. This finding could be attributable to the compensatory processes as response to MS injury and development. This hypothesis seems to be in good accordance with recent studies reporting adaptive responses during MS injuries and development. ${ }^{32} 33$

Another notable finding in this study is the decrease of the P600 amplitude in the patient group, located at left temporoparietal region. This finding seems-at least in part - to be in agreement with the study by Pelosi et al, ${ }^{7}$ even though this study differs substantially in some methodological dimensions such as the type of patients, the paradigm used, the stimulus type, recording sites, as well as the analysis time.

On the basis that the amplitude of P600 is considered as an index of the cost of reprocessing, the obtained results seem to be in agreement with psychophysiological studies, indicating that MS is accompanied by working memory deficits. ${ }^{5}$

Moreover, an alternative explanation concerning the significance of the observed differences of the P600 amplitude may be provided when taking into account the possible involvement of neurotransmitter systems. A review of psychopharmacological studies in the literature outlines the possible contribution of these mechanisms in relation to the particular recordings. Shelley et al found that the administration of droperidol (a dopamine antagonist) led to a significant attenuation of ERPs waveforms over the epochs $200 \mathrm{~ms}$ to 700 ms after warning stimulus, suggesting that changes of dopamine exert modulation over P600-like waveform. ${ }^{34}$ In this context, it is noteworthy that dopamine agonists such as bromocriptine, ${ }^{35}$ are used as therapeutic mean for MS.

In addition, it has been found that ACTH leads to an enhancement of the late positive waveform of ERPs. ${ }^{36} \mathrm{~A}$ very recent study provided evidence indicating that glucocorticoids (agents used as therapeutic means for MS), affect the P600 component. ${ }^{37}$ In particular, hydrocortisone administered to healthy controls led to augmentation of P600 amplitude over the frontal region in association with the exposure to novel stimuli, while the drug produced a reduction of P600 amplitude over the parietal area in response to repeated stimuli.

Concerning the memory performance, there was a significant difference between MS patients and normal controls, indicating that patients perform worse on the digit span tests. This finding is in agreement with a series of other related studies, ${ }^{23}{ }^{38}$ two of which concluded that the verbal memory functioning of MS patients is impaired.

It is worth noting that in this study no association was found between memory performance and observed differences concerning the P600 waveform. Possibly, this particular discrepancy may be credited to the ability of psychophysiological measures, such as the P600, to define the "endophenotype, ie the manifestation of a disorder via anomalies not observable by diagnostic interviews or traditional psychological measures." ${ }^{\prime 39}$

\section{Conclusions}

The results of this study should be interpreted with caution because of the following limitations; firstly, the post hoc assignation of psychological function to neurobiological activation is somewhat hypothetical and more specific techniques (for example, the inverse solution of the ERPs data to identify the underlying sources) $)^{40-42}$ investigating the role of a particular psychological process in the functional anatomy of MS are required. Secondly, taking into account the heterogeneity of MS patients, further studies are needed to specify the effects of illness subtypes and determine whether there is impairment in a task-specific manner or across tasks. Finally, in our sample most MS patients had long histories of drug treatment, mainly glucocorticoids. The effect of these somatic treatments on the obtained findings is unclear. Nevertheless, the obtained results could support the hypothesis that MS patients manifest abnormal aspects of "second pass parsing processes" of information as they are reflected by P600 elicited during a WM test. 


\section{Authors' affiliations}

C Sfagos, K K Kosma, D Vassilopoulos, Department of Neurology, Eginition Hospital, University of Athens, Athens, Greece

C C Papageorgiou, A D Rabavilas, Department of Psychiatry, Eginition Hospital, University of Athens

E Kodopadelis, N K Uzunoglu, Department of Electrical and Computer Engineering, National Technical University of Athens

A D Rabavilas, University Mental Health Research Institute, Athens

Competing interests: none declared.

\section{REFERENCES}

1 Foong J, Rozewicz L, Quaghebeur G, et al. Neuropsychological deficits in multiple sclerosis after acute relapse. J Neurol Neurosurg Psychiatry 1998:64:529-32.

2 DeSousa EA, Albert RH, Kalman B. Cognitive impairments in multiple sclerosis: a review. Am J Alzheimers Dis Other Demen 2002;17:23-9.

3 Amato MP, Ponziani G, Siracusa $G$, et al. Cognitive dysfunction in early-onset multiple sclerosis: a reappraisal after 10 years. Arch Neurol 2001;58:1602

4 Grigsby J, Ayarbe SD, Kravcisin N, et al. Working memory impairment among persons with chronic progressive multiple sclerosis. J Neurol 1994:241:125-31.

5 Sperling RA, Guttmann CR, Hohol M, et al. Regional magnetic resonance imaging lesion burden and cognitive function in multiple sclerosis: a longitudinal study. Arch Neurol 2001;58:115-21.

6 Diamond BJ, Deluca J, Kim H, et al. The question of disproportionate impairments in visual and auditory information processing in multiple sclerosis. J Clin Exp Neuropsychol 1997; 19:34-42.

7 Pelosi L, Geesken JM, Holly M, et al. Working memory impairment in early multiple sclerosis: evidence from an event-related potential study of patients with clinically isolated myelopathy. Brain 1997;120:2039-58.

8 Fabiani M, Gratton G, Coles M. Event-related potentials: methods, theory, and applications. In: Cacioppo J, Tassinary L, Bernston G, eds. Handbook of psychophysiology. New York: Cambridge University Press, 2000:53-84

9 Garcia-Larrea L, Cezanne-Bert G. Positive slow wave and working memory load: a study on the functional correlates of slow wave activity. Electroencephalogr Clin Neurophysiol 1998;108:260-73.

10 Friederici AD, Yves von Cramon D, Kotz SA. Language related brain potentials in patients and subcortical left hemisphere lesions. Brain 1999; 122:1033-47

11 Guillem F, Rougier A, Claverie B. Short- and long-delay intracranial ERP repetition effects dissociate memory systems in the human brain. Journal of Cognitive Neuroscience 1999; 1 1:437-58.

12 Baddeley A. Recent developments in working memory. Curr Opin Neurobiol 1998:8:234-8.

13 Collette $\mathbf{F}$, Van der Linden M. Brain imaging of the central executive component of working memory. Neurosci Biobehav 2002;26:105-25.

14 Miyake A, Shah P. Models of working memory. New York: Cambridge University Press, 1999.

15 Glassman R. A "theory of relativity" for cognitive elasticity of time and modality dimensions supporting constant working memory capacity: involvement of harmonics among ultradian clocks? Prog Neuropsychopharmacol Biol Psychiatry 2000;24: 163-82.

16 Guillem F, N'Kaoua B, Rougier A, et al. Intracranial topography of event-related potentials (N400/P600) elicited during a continuous recognition memory task. Psychophysiology 1995;32:382-92.

17 Filippi $M$, Rocca MA, Colombo B, et al. Functional magnetic resonance imaging correlates of fatigue in multiple sclerosis. Neuroimage 2002; 15:559-67.

18 Bermel RA, Bakshi R, Tjoa $C$, et al. Bicaudate ratio as a magnetic resonance imaging marker of brain atrophy in multiple sclerosis. Arch Neurol 2002;59:275-80.
19 Poser CM, Paty DW, Scheinberg L, et al. New diagnostic criteria for multiple sclerosis: guidelines for research protocols. Ann Neurol 1983;13:227-31.

20 Kurtzke JF. Rating neurologic impairment in multiple sclerosis: an expanded disability status scale (EDSS). Neurology 1983;33:1444-52.

21 Oldfield RC. The assessment and analysis of handedness. The Edinburgh Inventory. Neuropsychologia 1971;9:97-113.

22 Wechsler D. Manual for the Wechsler adult intelligence scale. New York: Psychological Corporation, 1955.

23 Papageorgiou C, Liappas I, Asvestas P, et al. Abnormal P600 in heroin addicts with prolonged abstinence elicited during a working memory test. Neuroreport 2001;12:1773-8.

24 Conklin H, Courtis C, Katsanis J, et al. Verbal working impairment in schizophrenia patients and their first-degree relatives: evidence from the digit span task. Am J Psychiatry 2000;157:275-7.

25 Perry W, Heaton RK, Potterat E, et al. Working memory in schizophrenia: transient, "online" storage versus executive functioning. Schizophr Bull 2001;27:157-76

26 Jasper $\mathbf{H}$. The ten-twenty electrode system of the international federation Electroencephalogr Clin Neurophysiol 1958;10:371-5.

27 Binder J. Functional magnetic resonance imaging: language mapping Neurosurg Clin N Am 1997;8:383-92.

28 Falkenstein $\mathbf{M}$, Hohnsbein J, Hoormann J. Effects of choice complexity on different subcomponents of the late positive complex of the event-related potential. Electroencephalogr Clin Neurophysiol 1994;92:148-60.

29 Nyberg L, Forkstam C, Petersson KM, et al. Brain imaging of human memory systems: between-systems similarities and within-system differences. Brain Res Cogn Brain Res 2002;13:281-92.

30 Landro NI, Rund BR, Lund A, et al. Honig's model of working memory and brain activation: an FMRI study. Neuroreport 2001:12:4047-54.

31 Lalonde R, Hannequin D. The neurobiological basis of time estimation and temporal order. Rev Neurosci 1999;10:151-73.

32 Filippi $M$. Linking structural, metabolic and functional changes in multiple sclerosis. Eur J Neurol 2001:8:291-7.

33 Tuohy VK, Yu M, Weinstock-Guttman B, et al. Diversity and plasticity of self recognition during the development of multiple sclerosis. J Clin Invest 1997;99:1682-90.

34 Shelley AM, Catts SV, Ward PB, et al. The effect of decreased catecholamine transmission on ERP indices of selective attention. Neuropsychopharmacology 1997;16:202-10

35 McMurray RW. Bromocriptine in rheumatic and autoimmune diseases. Semin Arthritis Rheum 2001;31:21-32.

36 Smolnik R, Perras B, Molle M, et al. Event-related brain potentials and working memory function in healthy humans after single-dose and prolonged intranasal administration of adrenocorticotropin 4-10 and desacetyl-alpha-melanocyte stimulating hormone. J Clin Psychopharmacol 2000;20:445-54

37 Monk CS, Nelson CA. The effects of hydrocortisone on cognitive and neural function: a behavioral and event-related potential investigation. Neuropsychopharmacology 2002;26:505-19.

38 Camp SJ, Stevenson VL, Thompson AJ, et al. Cognitive function in primary progressive and transitional progressive multiple sclerosis: a controlled study with MRI correlates. Brain 1999;122:1341-8.

39 Klein DN, Anderson RL. The behavioral high-risk paradigm in the mood disorders. In: Miller GA, ed. The behavioral high-risk paradigm in psychopathology. New York: Springer-Verlag; 1995:199-221.

40 Pascual-Marqui RD, Michel CM, Lehmann D. Segmentation of brain electrical activity into microstates: model estimation and validation. IEEE Trans Biomed Eng 1995;42:658-65.

41 Lehmann D, Skrandies W. Spatial analysis of evoked potentials in man - a review. Prog Neurobiol 1984;23:227-50.

42 Michel CM, Thut G, Morand S, et al. Electric source imaging of human brain functions. Brain Res Brain Res Rev 2001;36:108-18. 